\title{
Dislocation density reduction via lateral epitaxy in selectively grown GaN structures
}

\author{
Tsvetanka S. Zheleva, Ok-Hyun Nam, Michael D. Bremser, and Robert F. Davis \\ Department of Materials Science and Engineering, North Carolina State University, Raleigh, North Carolina \\ 27695-7907
}

(Received 23 June 1997; accepted for publication 22 August 1997)

\begin{abstract}
The microstructure and the lateral epitaxy mechanism of formation of homoepitaxially and selectively grown $\mathrm{GaN}$ structures within windows in $\mathrm{SiO}_{2}$ masks have been investigated by transmission electron microscopy (TEM) and scanning electron microscopy. The structures were produced by organometallic vapor phase epitaxy for field emission studies. A GaN layer underlying the $\mathrm{SiO}_{2}$ mask provided the crystallographic template for the initial vertical growth of the GaN hexagonal pyramids or striped pattern. The $\mathrm{SiO}_{2}$ film provided an amorphous stage on which lateral growth of the GaN occurred and possibly very limited compliancy in terms of atomic arrangement during the lateral growth and in the accommodation of the mismatch in the coefficients of thermal expansion during cooling. Observations with TEM show a substantial reduction in the dislocation density in the areas of lateral growth of the $\mathrm{GaN}$ deposited on the $\mathrm{SiO}_{2}$ mask. In many of these areas no dislocations were observed. (C) 1997 American Institute of Physics. [S0003-6951(97)00843-7]
\end{abstract}

Group III nitrides are being extensively investigated for microelectronic and optoelectronic device applications due to their wide direct band gap and good thermal, chemical, and mechanical stability. Two examples of the numerous published reviews and compilations of this research are given in Refs. 1 and 2. The negative electron affinity of $\mathrm{AlN}^{3}$ and the low positive electron affinity of $\mathrm{GaN}^{4,5}$ also make them promising candidate materials for field emitters. ${ }^{5,6}$ The selective growth of $\mathrm{GaN}$ hexagonal pyramids and stripes on patterned $\mathrm{GaN}(0001)$ films on sapphire $e^{4,7,8}$ and $6 \mathrm{H}-$ $\mathrm{SiC}(0001)$ substrates $^{5,9}$ have been reported. The defect free microstructure of selectively grown $\mathrm{GaN}$ related to the lateral epitaxy formation mechanism is reported in the present study.

Selective homoepitaxial growth of GaN pyramids and stripes has been achieved on circular patterned GaN/AlN/ $6 \mathrm{H}-\mathrm{SiC}(0001)$ multilayered structures having a top $\mathrm{SiO}_{2}$ layer containing circular windows with 5 and $10 \mu \mathrm{m}$ diameters for the pyramids, and 3 and $5 \mu \mathrm{m}$ striped windows for the GaN stripes. The nitride films were grown in a cold wall, vertical, pancake-type, rf inductively heated organometallic vapor phase epitaxy (OMVPE) system. The experimental growth parameters of the films are described elsewhere. ${ }^{10}$ The thicknesses of the AlN buffer layer and the subsequently grown GaN layer were $1000 \AA$ and $1.75 \mu \mathrm{m}$, respectively. The $\mathrm{SiO}_{2}$ layers were deposited via rf sputtering or low pressure chemical vapor deposition. The parameters for the selective OMVPE growth of the GaN have also been reported previously. ${ }^{5,9}$ In the present research the morphology, defect microstructure, and formation mechanism of the pyramids and stripes have been investigated by scanning electron microscopy (SEM) (JEOL 6400 FE) and transmission electron microscopy (TEM) (TOPCON 002B, 200 kV). Crosssectional samples for TEM have been prepared using standard procedures involving the sequence of grinding, polishing, and ion milling. All of the presented TEM micrographs are in bright field imaging conditions.

Each of the pyramids has six (1 $\overline{101})$ side facets as ob- served via SEM and shown in Fig. 1. The final size of the base of the GaN pyramids as well as their height depend on the window-to-mask area ratios. Reduction of this ratio leads to an increase in the average lateral width under the same growth conditions. ${ }^{9}$

Cross-sectional TEM from a pyramid array is shown in Fig. 2. This figure reveals that the pyramids grow with sharp closed tops and as single crystals, as revealed from the selected area diffraction pattern shown as an inset. The variety of facet angles evident from the low magnification TEM micrograph reflects the fact that during the TEM sample preparation the cut is made not in a straight line crossing one pyramid row only, but at an angle to a particular row and cutting different slices through the pyramids which have different heights and angles with respect to their normal. Observations with TEM also reveal that the GaN pyramids extend laterally at dimensions larger than the diameters of the windows. A low magnification TEM micrograph of a portion of one GaN pyramid in the [11 $\overline{20}]$ orientation is shown in Fig. 3(a). Representative distributions of the dislocations within the pyramid and the underlying GaN film are clearly observed. The defects in the initial GaN film are threading

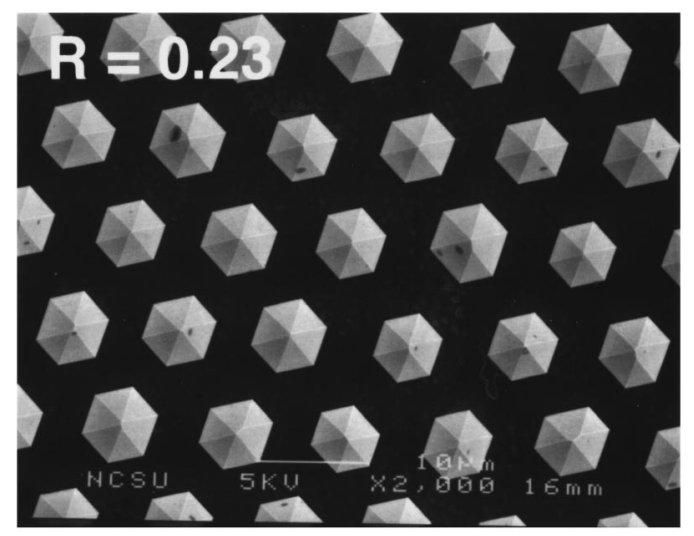

FIG. 1. SEM picture of a pyramid array grown at 0.23 ratio of the windowto-mask areas. 


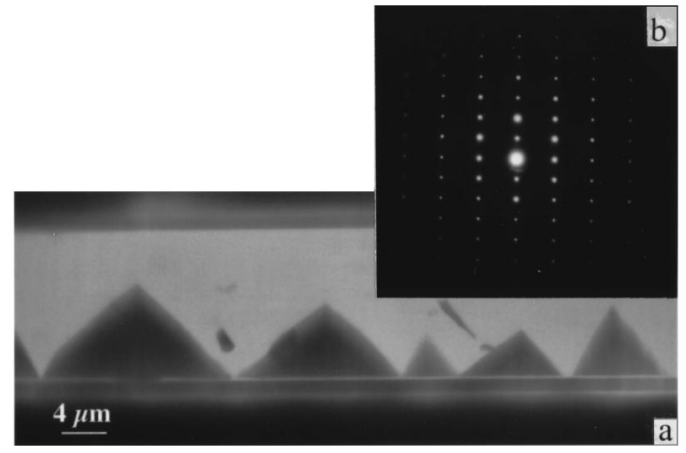

FIG. 2. Low magnification cross-sectional TEM of GaN pyramid array in $[11 \overline{2} 0]$ orientation. The inset is a selected area diffraction pattern from a pyramid revealing its single crystal character.

segments of dislocations perpendicular or nearly perpendicular to the interface plane and short dislocation half loops near the GaN/AlN interface. It is important to note that the dislocations extend into each pyramid only above the window areas. Thus, the microstructure of each pyramid is characterized by two distinct regions, denoted $\mathrm{A}$ and $\mathrm{B}$ and shown pictorially in Fig. 3(a) and schematically in Fig. 3(b). Region A, located above the window area, and corresponding to the vertical growth, contains dislocations at a density comparable with that of the underlying GaN film within $\sim 3 \mu \mathrm{m}$ distance from the GaN/GaN homoepitaxial interface. Most of the extended dislocations propagate throughout the $\mathrm{GaN}$ film from the GaN/AlN and AlN/6H-SiC interfaces. The main cause of the generation of these defects is the mismatch in the lattice parameters at these interfaces. The final distribution of the dislocations is determined by the differences in both lattice parameters and coefficients of thermal expansion among these phases. The dislocation density diminishes within a pyramidal volume which ends at approximately one third of the pyramid height; no dislocations were observed at higher elevations in the pyramid structure.

Region $\mathrm{B}$ of the pyramid above the $\mathrm{SiO}_{2}$ mask area surrounding the window, and corresponding to the lateral growth, is nearly free of observable defects. Very few short edge-on dislocation segments (D) parallel to the interface plane and few overlapping stacking faults (SF) in the vicinity of the $\mathrm{GaN}$ pyramid/ $\mathrm{SiO}_{2}$ interface were observed. The high resolution TEM from the top and from the (1101) sidewalls of the pyramids again revealed material regions essentially free of dislocations.

Analysis with TEM revealed similar results about the microstructure of selectively grown $\mathrm{GaN}$ striped pattern and namely substantial reduction in dislocation density in the areas of lateral growth of GaN. The low magnification TEM micrograph from a selectively grown $\mathrm{GaN}$ stripe shown in Fig. 4 clearly reveals the difference in the dislocation density in the areas with vertical growth $\left(\sim 10^{8}-10^{9} / \mathrm{cm} 2\right)$ and those with lateral growth over the $\mathrm{SiO}_{2}$ mask (defect free). In our best samples we were able to reveal dislocation free regions of dimensions $4.5 \mu \mathrm{m}$ along the interface line or [1100] direction $\times 9.3 \mu \mathrm{m}$ along [0001] or the normal with respect to the film surface $\times \sim 0.1 \mu \mathrm{m}$ within the sample thickness or along the [1120] direction.

The formation mechanism of the selectively grown $\mathrm{GaN}$
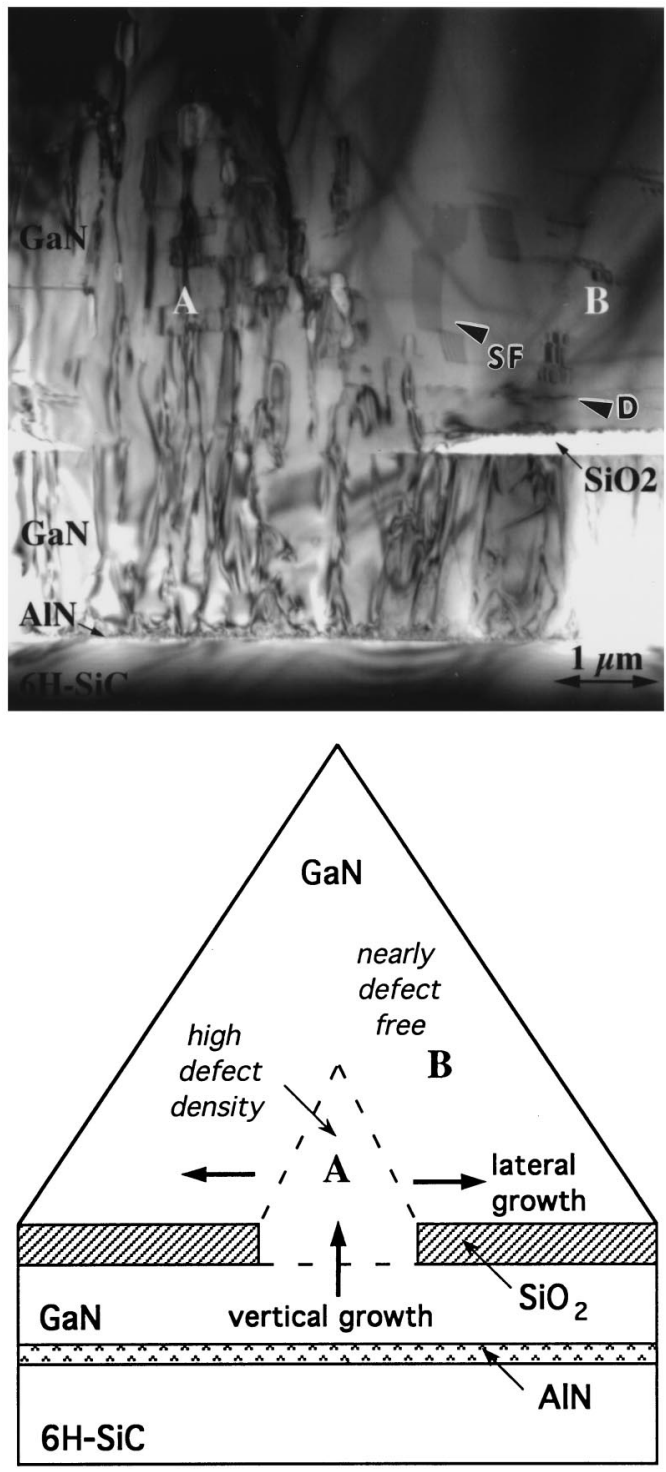

FIG. 3. (a) Microstructure of selectively grown GaN pyramid in [11 $\overline{2} 0]$ orientation of the foil. Two distinct regions are observed: region A, located above the window in the $\mathrm{SiO}_{2}$ and having a high dislocation density; and region $\mathrm{B}$ located above the mask with very few dislocations (D), with lines parallel to the interface plane and a low concentration of overlapping stacking faults (SF) close to the $\mathrm{GaN} / \mathrm{SiO}_{2}$ interface. (b) A schematic of the typical microstructure and lateral epitaxy mechanism of the selectively grown pyramid.

structures in this study is lateral epitaxy. The two main stages of this mechanism are: (i) vertical growth and (ii) lateral growth. ${ }^{11}$ During the first stage the deposited GaN grows selectively within the $\mathrm{GaN}$ windows more rapidly than it grows over the surrounding $\mathrm{SiO}_{2}$ mask region due to the much higher sticking coefficient, $s$, of the Ga adatoms on the GaN surface $(s=1)$ than on $\mathrm{SiO}_{2}$ mask $(s \sim 0)$. This is the expected result as the $\mathrm{SiO}_{2}$ bond strength is $799.6 \mathrm{~kJ} / \mathrm{mol}$ and much higher than that of $\mathrm{Si}-\mathrm{N}(439 \mathrm{~kJ} / \mathrm{mol}), \mathrm{Ga}-\mathrm{N}(103$ $\mathrm{kJ} / \mathrm{mol})$, and $\mathrm{Ga}-\mathrm{O}(353.6 \mathrm{~kJ} / \mathrm{mol}) .{ }^{12}$ Thus it would be unlikely for $\mathrm{Ga}$ or $\mathrm{N}$ adatoms to bond to the $\mathrm{SiO}_{2}$ surface in numbers and for a time sufficient to cause $\mathrm{GaN}$ nuclei to form. They would either evaporate or diffuse along the surface to the opening in the mask or to the vertical GaN surfaces which have emerged. During the second stage the GaN grows simultaneously both vertically and laterally over the 


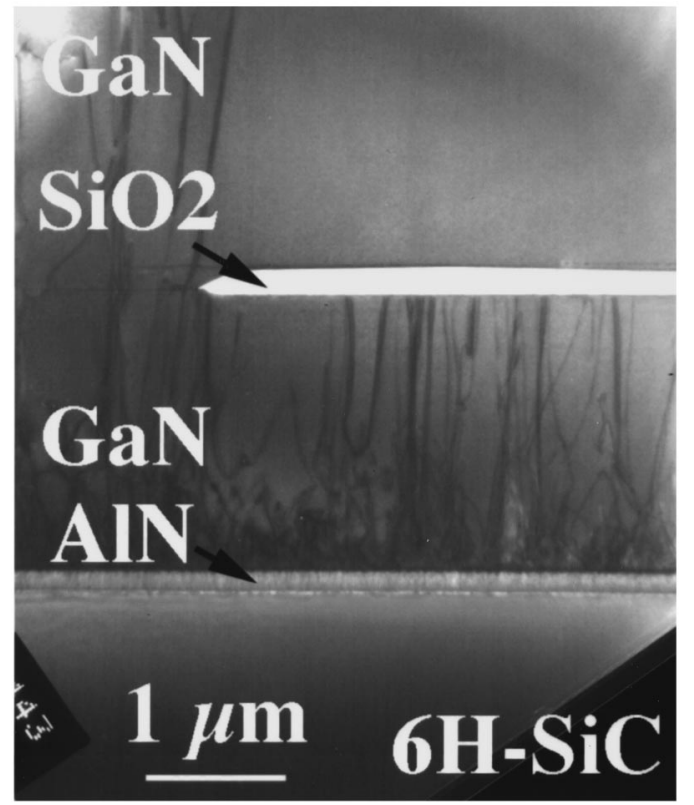

FIG. 4. Cross-sectional TEM micrograph in $[11 \overline{2} 0]$ orientation from a selectively grown $\mathrm{GaN}$ stripe revealing the difference in dislocation density in the areas with vertical growth $\left(10^{8}-10^{9} / \mathrm{cm}^{2}\right)$ and those with lateral growth over the $\mathrm{SiO}_{2}$ mask (defect free).

mask from the material which emerges over the windows.

Surface diffusion of $\mathrm{Ga}$ and $\mathrm{N}$ on the $\mathrm{SiO}_{2}$ may play a minor role in $\mathrm{GaN}$ selective growth; however, the major source of material is derived from the gas phase. This has been demonstrated by the fact that an increase in the TEG flow rate causes the growth rate of the (0001) top facets to develop faster than the (1101) side facets and thus controls the lateral growth. ${ }^{89}$

The laterally grown $\mathrm{GaN}$ is bonded to the underlying $\mathrm{SiO}_{2}$ sufficiently strongly that it does not break away on cooling. However, lateral cracking within the $\mathrm{SiO}_{2}$ is commonly but not universally observed in the samples as a result of thermal stresses generated on cooling. The nature of the bonding of the laterally grown $\mathrm{GaN}$ with the $\mathrm{SiO}_{2}$, i.e., mechanical, chemical, or both is not known at this time. The viscosity $(\rho)$ of the $\mathrm{SiO}_{2}$ at the growth temperature of $1050{ }^{\circ} \mathrm{C}$ is $\sim 10^{15.5}$ poise which is one order of magnitude greater than the strain point $\left(\sim 10^{14.5}\right.$ poise $)$ where stress relief in a bulk amorphous material occurs within approximately six hours. ${ }^{13}$ Thus the $\mathrm{SiO}_{2}$ provides limited compliancy on cooling. As the atomic arrangement on the amorphous $\mathrm{SiO}_{2}$ surface is quite different from that on the $\mathrm{GaN}$ surface, chemical bonding would occur only when appropriate pairs of atoms are in close proximity. Extremely small relaxations of the $\mathrm{Si}, \mathrm{O}, \mathrm{Ga}$, and $\mathrm{N}$ atoms on the respective surfaces and/or within the bulk of the $\mathrm{SiO}_{2}$ may accommodate the $\mathrm{GaN}$ and cause it to bond to the oxide.

Finally, the coalescence of the laterally growing volumes results in nearly defect free regions, as shown in Fig. 5. Detailed studies of the selective growth and the lateral epitaxy mechanism of the latter structures will be presented elsewhere.

In summary, significant reduction in the dislocation density is achieved via lateral epitaxy in selectively grown $\mathrm{GaN}$ pyramids and striped structures deposited within windows

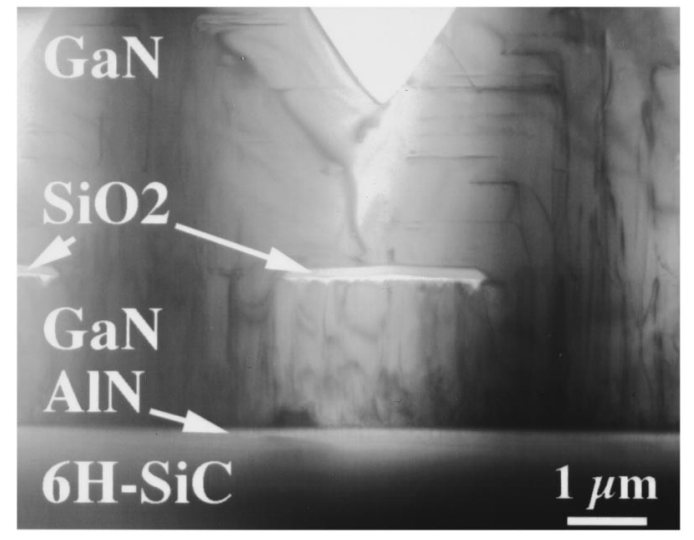

FIG. 5. Microstructure of a region of coalescence of two laterally grown areas of $\mathrm{GaN}$ stripes in [11 $\overline{2} 0]$ orientation of the foil. Please note the change of the direction of the dislocation lines with the growth direction and the fact that the dislocation lines are predominantly parallel to the growth direction-initially_parallel to [0001] during the vertical growth and thereafter parallel to [1100] direction, thus following the lateral growth.

contained in $\mathrm{SiO}_{2}$ mask on GaN/AlN/6H-SiC heterostructure. Analysis with TEM reveals that lateral epitaxy over the amorphous $\mathrm{SiO}_{2}$ provided the formation mechanism for large volume percentages of nearly defect free single crystal GaN. Investigations regarding additional factors which control the growth rate and the optimization of the growth conditions are ongoing, as this process route has the potential as an alternative growth scheme to the conventional epitaxial growth of high quality GaN layered structures.

The authors acknowledge the support of the Office of Naval Research via Contract No. N00014-96-1-0765 and Cree Research, Inc. for the SiC wafers. R. Davis acknowledges personal support from the Kobe Steel Ltd. Professorship.

${ }^{1}$ H. Morkoç, S. Strite, G. Gao, M. Lin, B. Sverdlov, and M. Burns, J. Appl. Phys. 76, 1363 (1994).

${ }^{2}$ F. A. Ponce, T. D. Moustakas, I. Akasaki, and B. A. Monemar, editors, III-N Nitrides, Materials Research Society Symposium Proceedings, 1997 (unpublished), Vol. 449.

${ }^{3}$ R. Nemanich, M. Bendjamin, S. Boseman, M. Bremser, S. King, B. Ward, R. F. Davis, B. Chen, Z. Zhang, and J. Bernholc, Materials Research Society Fall Meeting, Boston, November, 1995.

${ }^{4}$ R. Underwood, D. Kapolnek, B. Keller, S. Keller, S. DenBaars, and U. Mishra, Topical Workshop on Nitrides, Nagoya, Japan, September, 1995.

${ }^{5}$ O. H. Nam, M. Bremser, B. Ward, R. Nemanich, and R. F. Davis, Materials Research Society Fall Meeting, Boston, November, 1996.

${ }^{6}$ R. Underwood, D. Kapolnek, B. Keller, S. Keller, S. DenBaars, and U. Mishra, Solid-State Electron. 41, 243 (1997).

${ }^{7}$ Y. Kato, S. Kitamura, K. Hiramatsu, and N. Sawaki, J. Cryst. Growth 144, 133 (1994).

${ }^{8}$ S. Kitamura, K. Hiramatsu, and N. Sawaki, Jpn. J. Appl. Phys., Part 1 34, 1184 (1985).

${ }^{9}$ O. H. Nam, M. Bremser, B. Ward, R. Nemanich, and R. F. Davis, Jpn. J. Appl. Phys., Part 1 36, L532 (1997).

${ }^{10}$ T. W. Weeks, Jr., M. Bremser, K. Ailey, E. Carlson, W. Perry, E. Piner, N. ElMasry, and R. F. Davis, J. Mater. Res. 11, 1011 (1996).

${ }^{11}$ E. I. Givargizov, Oriented Crystallization on Amorphous Substrates (Plenum, New York, 1991), pp. 221-264.

${ }^{12}$ CRC Handbook of Chemistry and Physics, 72nd ed., edited by D. Lide (CRC, Boca Raton, 1991-1992).

${ }^{13}$ A. Varshneya, Fundamentals of Inorganic Glasses (Academic, New York, 1994), pp. 183-204. 